Supporting Information

\title{
Insights into the Loop at E-selectin Binding Site: from Open to Close Conformation
}

\author{
Xiaocong Wang, Lihua Bie, Junwen Fei, Jun Gao* \\ Hubei Key Laboratory of Agricultural Bioinformatics, College of Informatics, Huazhong \\ Agricultural University, Wuhan, Hubei, 430070, China
}

*E-mail: gaojun@mail.hzau.edu.cn 
Table S1. Essential hydrogen bond interactions for the open and close loop conformations in the MD simulation.

\begin{tabular}{|c|c|c|c|c|c|c|c|}
\hline \multicolumn{2}{|c|}{ Ligand } & \multicolumn{2}{|c|}{ E-selectin } & \multirow{2}{*}{$\frac{\text { open conformation }}{2.9 \pm 0.2(55)^{\mathrm{a}}}$} & \multicolumn{2}{|c|}{ E-selectin } & \multirow{2}{*}{$\frac{\text { close conformation }}{2.8 \pm 0.3(58)}$} \\
\hline & O1 & R97 & $\mathrm{N} \eta 1$ & & Y48 & $\mathrm{O \eta}$ & \\
\hline \multirow[t]{2}{*}{ Neu5Ac } & O1 & R97 & $\mathrm{N} \eta 2$ & $2.9 \pm 0.2(64)$ & R97 & $\mathrm{N} \varepsilon$ & $3.0 \pm 0.2(51)$ \\
\hline & O4 & & & & E98 & $\mathrm{O} \varepsilon$ & $2.8 \pm 0.2(29)$ \\
\hline \multirow{5}{*}{$\begin{array}{c}\text { Core-2 } \\
\text { Gal }\end{array}$} & $\mathrm{O} 3$ & & & & R97 & $\mathrm{N} \eta 2$ & $3.1 \pm 0.2(47)$ \\
\hline & $\mathrm{O} 4$ & & & & Y94 & $\mathrm{O \eta}$ & $3.0 \pm 0.2(40)$ \\
\hline & $\mathrm{O} 4$ & & & & R97 & $\mathrm{N} \eta 2$ & $3.0 \pm 0.2(38)$ \\
\hline & O6 & E92 & $\mathrm{O} \varepsilon^{\mathrm{b}}$ & $2.7 \pm 0.2(83)$ & E92 & $\mathrm{O} \varepsilon$ & $2.8 \pm 0.2(97)$ \\
\hline & O6 & Y94 & $\mathrm{O \eta}$ & $2.9 \pm 0.2(55)$ & Y94 & On & \\
\hline \multirow[t]{3}{*}{ GlcNAc } & $\mathrm{O} 2 \mathrm{~N}$ & K111 & $\mathrm{N} \zeta$ & $2.9 \pm 0.2(72)$ & & & $2.9 \pm 0.1(65)$ \\
\hline & $\mathrm{O} 2$ & N83 & $\mathrm{N} \delta$ & -- & E88 & $\mathrm{O} \varepsilon$ & $2.8 \pm 0.2(30)$ \\
\hline & $\mathrm{O} 2$ & E107 & $\mathrm{O} \varepsilon^{\mathrm{b}}$ & $2.8 \pm 0.1(92)$ & & & \\
\hline \multirow[t]{3}{*}{ Fuc } & $\mathrm{O} 3$ & N105 & $\mathrm{N} \delta$ & $3.1 \pm 0.1(87)$ & N105 & $\mathrm{N} \delta$ & $3.1 \pm 0.2(80)$ \\
\hline & $\mathrm{O} 3$ & E107 & $\mathrm{O} \varepsilon^{\mathrm{b}}$ & $2.6 \pm 0.1(100)$ & E107 & $\mathrm{O} \varepsilon$ & $2.6 \pm 0.1(85)$ \\
\hline & $\mathrm{O} 4$ & E80 & $\mathrm{O} \varepsilon^{\mathrm{b}}$ & $2.8 \pm 0.2(100)^{\mathrm{c}}$ & E80 & $\mathrm{O} \varepsilon$ & $2.8 \pm 0.2(100)^{\mathrm{c}}$ \\
\hline
\end{tabular}

${ }^{a}$ Percentage (\%) based on a distance between non-hydrogen atoms of less than $3.5 \AA$. Distance in A..

${ }^{b}$ When multiple hydrogen bonds are formed between two heavy atoms through different hydrogens, the occupancy of the interaction listed is the sum of all the individual hydrogen bonds and the distance is the average of all the individual hydrogen bonds.

${ }^{c}$ The occupancy of the interactions between two heavy atoms, calculated as the sum of all the individual hydrogen bonds through different hydrogens, is greater than $100 \%$. 
Table S2. Essential hydrogen bond interactions in ASMD simulation starting from co-crystal complex.

\begin{tabular}{|c|c|c|c|c|c|c|c|c|c|c|c|}
\hline \multicolumn{2}{|c|}{ E-selectin } & \multicolumn{2}{|c|}{$\mathrm{sLe}^{\mathrm{x}}$} & $0 \sim 1 \AA$ & $1 \sim 2 \AA$ & $2 \sim 3 \AA$ & $3 \sim 4 \AA$ & $4 \sim 5 \AA$ & $5 \sim 6 \AA$ & $6 \sim 7 \AA$ & $7 \sim 8 \AA$ \\
\hline \multirow{4}{*}{ Neu5Ac } & $\mathrm{O}^{\mathrm{b}}$ & Y48 & On & $3.0 \pm 0.2(100)^{\mathrm{a}, \mathrm{c}}$ & $3.0 \pm 0.2(100)^{\mathrm{c}}$ & & & & & & \\
\hline & $\mathrm{O} 1^{\mathrm{b}}$ & R97 & $\mathrm{N} \varepsilon$ & $2.9 \pm 0.2(95)$ & $2.9 \pm 0.1(100)$ & $3.0 \pm 0.2(83)$ & & & & & \\
\hline & $\mathrm{O} 1^{\mathrm{b}}$ & R97 & $\mathrm{N} \eta 2$ & $2.9 \pm 0.2(94)$ & $2.9 \pm 0.2(93)$ & $2.9 \pm 0.2(96)$ & $2.9 \pm 0.2(92)$ & $3.0 \pm 0.2(89)$ & & & \\
\hline & $\mathrm{O} 1^{\mathrm{b}}$ & Q85 & $\mathrm{N} \varepsilon$ & & & & & & & & $3.0 \pm 0.2(41)$ \\
\hline \multirow{7}{*}{$\begin{array}{c}\text { Core-2 } \\
\text { Gal }\end{array}$} & $\mathrm{O} 3$ & R97 & $\mathrm{N \eta} 2$ & $3.2 \pm 0.2(46)$ & $3.2 \pm 0.2(57)$ & $3.2 \pm 0.2(37)$ & & & & & \\
\hline & $\mathrm{O} 4$ & Y94 & $\mathrm{O \eta}$ & $3.0 \pm 0.2(45)$ & $3.0 \pm 0.2(86)$ & $3.0 \pm 0.2(46)$ & & & & & \\
\hline & O6 & E92 & $\mathrm{O} \varepsilon^{\mathrm{b}}$ & $2.8 \pm 0.1(123)$ & $2.8 \pm 0.1(100)^{\mathrm{c}}$ & $2.7 \pm 0.2(68)$ & & & & & \\
\hline & O6 & Y94 & $\mathrm{O \eta}$ & $2.9 \pm 0.2(83)$ & $2.9 \pm 0.2(93)$ & $2.8 \pm 0.2(70)$ & & & & & \\
\hline & O6 & N105 & $\mathrm{N} \delta$ & & & $3.2 \pm 0.2(26)$ & & & & & \\
\hline & O6 & E107 & $\mathrm{O} \varepsilon^{\mathrm{b}}$ & & & $2.9 \pm 0.2(40)$ & $2.8 \pm 0.1(93)$ & $2.9 \pm 0.2(70)$ & & & \\
\hline & O6 & Q85 & $\mathrm{N} \varepsilon$ & & & & & & $3.1 \pm 0.2(53)$ & $3.0 \pm 0.2(31)$ & \\
\hline \multirow{2}{*}{ GlcNAc } & $\mathrm{O} 2 \mathrm{~N}$ & Q85 & $\mathrm{N} \varepsilon$ & $2.9 \pm 0.2(65)$ & $2.9 \pm 0.2(50)$ & $2.9 \pm 0.2(92)$ & $2.9 \pm 0.2(93)$ & $2.9 \pm 0.2(38)$ & & & \\
\hline & $\mathrm{O} 3$ & Q85 & $\mathrm{N} \varepsilon$ & & & & & & $3.2 \pm 0.2(35)$ & & \\
\hline \multirow{13}{*}{ Fuc } & $\mathrm{O} 2$ & E88 & $\mathrm{O} \varepsilon^{\mathrm{b}}$ & $2.8 \pm 0.2(111)$ & $2.9 \pm 0.2(100)^{\mathrm{c}}$ & $3.0 \pm 0.2(100)^{\mathrm{c}}$ & $2.8 \pm 0.2(86)$ & $2.8 \pm 0.2(50)$ & & & \\
\hline & $\mathrm{O} 3$ & N105 & $\mathrm{N} \delta$ & $3.3 \pm 0.1(52)$ & & & & & & & \\
\hline & $\mathrm{O} 3$ & E107 & $\mathrm{O} \varepsilon^{\mathrm{b}}$ & $2.8 \pm 0.2(99)$ & $2.8 \pm 0.2(100)$ & $2.7 \pm 0.1(100)$ & $2.7 \pm 0.1(100)$ & $2.7 \pm 0.1(44)$ & & & \\
\hline & O4 & E80 & $\mathrm{O} \varepsilon^{\mathrm{b}}$ & $2.8 \pm 0.2(100)^{c}$ & $2.8 \pm 0.1(100)^{\mathrm{c}}$ & $2.8 \pm 0.1(100)^{\mathrm{c}}$ & $2.9 \pm 0.1(100)^{\mathrm{c}}$ & $2.8 \pm 0.1(67)$ & & & \\
\hline & $\mathrm{O} 4$ & N82 & $\mathrm{N} \delta$ & $2.9 \pm 0.2(39)$ & $2.8 \pm 0.1(47)$ & & & $2.8 \pm 0.1(25)$ & & & \\
\hline & $\mathrm{O} 4$ & R84 & $\mathrm{N} \varepsilon$ & $3.0 \pm 0.2(44)$ & & & & & & & \\
\hline & $\mathrm{O} 4$ & R84 & $\mathrm{N} \eta 2$ & $3.1 \pm 0.2(50)$ & & & & & $3.0 \pm 0.2(30)$ & & \\
\hline & $\mathrm{O} 3$ & N82 & $\mathrm{N} \delta$ & & & & & $3.1 \pm 0.2(32)$ & & & \\
\hline & $\mathrm{O} 3$ & R84 & $\mathrm{N} \varepsilon$ & & & & & & $2.9 \pm 0.2(33)$ & & \\
\hline & $\mathrm{O} 2$ & Q85 & $\mathrm{N} \varepsilon$ & & & & & & $3.0 \pm 0.2(86)$ & $3.0 \pm 0.2(21)$ & \\
\hline & $\mathrm{O} 2$ & $\mathrm{R} 84$ & $\mathrm{~N} \eta 1$ & & & & & & & $3.0 \pm 0.2(24)$ & $3.0 \pm 0.2(34)$ \\
\hline & $\mathrm{O} 2$ & R84 & $\mathrm{N} \eta 2$ & & & & & & & & $2.9 \pm 0.2(47)$ \\
\hline & $\mathrm{O} 3$ & R84 & $\mathrm{N} \eta 1$ & & & & & & & & $2.9 \pm 0.2(26)$ \\
\hline
\end{tabular}

${ }^{a}$ Percentage (\%) based on a distance between non-hydrogen atoms of less than $3.5 \AA$. Distance in $\AA$.

${ }^{b}$ When multiple hydrogen bonds are formed between two heavy atoms through different hydrogens, the occupancy of the interaction listed is the sum of all the individual hydrogen bonds and the distance is the average of all the individual hydrogen bonds.

${ }^{c}$ The occupancy of the interactions between two heavy atoms, calculated as the sum of all the individual hydrogen bonds through different hydrogens, is greater than $100 \%$. 

Table S3. Essential hydrogen bond interactions in ASMD simulation starting from soak complex.

\begin{tabular}{|c|c|c|c|c|c|c|c|c|c|c|c|}
\hline \multicolumn{2}{|c|}{ E-selectin } & \multicolumn{2}{|c|}{$\mathrm{sLe}^{\mathrm{x}}$} & \multirow[t]{2}{*}{$0 \sim 1 \AA$} & \multirow{2}{*}{$\frac{1 \sim 2 \AA}{2.9 \pm 0.2(26)^{\mathrm{a}}}$} & \multirow[t]{2}{*}{$2 \sim 3 \AA$} & \multirow[t]{2}{*}{$3 \sim 4 \AA$} & \multirow[t]{2}{*}{$4 \sim 5 \AA$} & \multirow[t]{2}{*}{$5 \sim 6 \AA$} & \multirow[t]{2}{*}{$6 \sim 7 \AA$} & \multirow[t]{2}{*}{$7 \sim 8 \AA$} \\
\hline & $\mathrm{O} 1^{\mathrm{b}}$ & R97 & $\mathrm{N} \eta 2$ & & & & & & & & \\
\hline \multirow[t]{2}{*}{ Neu5Ac } & $\mathrm{O} 1^{\mathrm{b}}$ & K111 & $\mathrm{N} \zeta$ & & & & & $2.9 \pm 0.2(100)^{\mathrm{c}}$ & $2.9 \pm 0.2(73)$ & & \\
\hline & $\mathrm{O} 1^{\mathrm{b}}$ & N82 & $\mathrm{N} \delta$ & & & & & & & $2.9 \pm 0.2(82)$ & $2.9 \pm 0.2(90)$ \\
\hline \multirow{10}{*}{$\begin{array}{c}\text { Core-2 } \\
\text { Gal }\end{array}$} & O6 & E107 & $\mathrm{O} \varepsilon^{\mathrm{b}}$ & $2.9 \pm 0.2(87)$ & $2.9 \pm 0.3(87)$ & $2.9 \pm 0.3(80)$ & $2.9 \pm 0.2(92)$ & & & & \\
\hline & O6 & K111 & $\mathrm{N} \zeta$ & $3.1 \pm 0.2(22)$ & $3.1 \pm 0.2(21)$ & $3.0 \pm 0.2(41)$ & & & & & \\
\hline & $\mathrm{O} 2$ & N83 & $\mathrm{N} \delta$ & $3.2 \pm 0.2(42)$ & $3.2 \pm 0.2(53)$ & $3.2 \pm 0.2(34)$ & $3.1 \pm 0.2(37)$ & & & & \\
\hline & $\mathrm{O} 3$ & N83 & $\mathrm{N} \delta$ & $2.9 \pm 0.1(100)$ & $3.0 \pm 0.2(85)$ & $2.9 \pm 0.1(100)$ & $2.9 \pm 0.1(98)$ & $3.0 \pm 0.2(25)$ & & & \\
\hline & $\mathrm{O} 3$ & E107 & $\mathrm{O} \varepsilon^{\mathrm{b}}$ & $2.9 \pm 0.2(100)^{\mathrm{c}}$ & $2.8 \pm 0.2(100)^{\mathrm{c}}$ & $2.8 \pm 0.2(100)^{\mathrm{c}}$ & $2.8 \pm 0.3(91)$ & & & & \\
\hline & $\mathrm{O} 4$ & E80 & $\mathrm{O} \varepsilon^{\mathrm{b}}$ & $2.7 \pm 0.1(90)$ & $2.7 \pm 0.1(97)$ & $2.7 \pm 0.1$ (99) & $2.7 \pm 0.2(89)$ & & & & \\
\hline & $\mathrm{O} 4$ & N82 & $\mathrm{N} \delta$ & $3.4 \pm 0.1$ (27) & $3.4 \pm 0.1$ (43) & $3.3 \pm 0.1$ (47) & $3.3 \pm 0.2(48)$ & $3.0 \pm 0.1(26)$ & & & \\
\hline & $\mathrm{O} 4$ & D105 & $\mathrm{O} \delta$ & $3.0 \pm 0.2(24)$ & & & & & & & \\
\hline & O5 & N82 & $\mathrm{N} \delta$ & $3.1 \pm 0.2(76)$ & $3.1 \pm 0.2(67)$ & $3.1 \pm 0.2(51)$ & $3.1 \pm 0.2(28)$ & & & & \\
\hline & $\mathrm{O} 3$ & Q85 & $\mathrm{O} \varepsilon^{\mathrm{b}}$ & & & & & & $2.7 \pm 0.1(40)$ & & \\
\hline \multirow[t]{9}{*}{ Fuc } & $\mathrm{O} 4$ & N82 & $\mathrm{O}$ & & & & & $2.9 \pm 0.2(23)$ & $2.8 \pm 0.2(99)$ & $2.8 \pm 0.1(34)$ & \\
\hline & $\mathrm{O} 2$ & Q85 & $\mathrm{N} \varepsilon$ & & & & & & $3.0 \pm 0.2(39)$ & & \\
\hline & $\mathrm{O} 4$ & $\mathrm{R} 84$ & $\mathrm{~N} \varepsilon$ & & & & & & $3.0 \pm 0.2(23)$ & & \\
\hline & $\mathrm{O} 4$ & $\mathrm{R} 84$ & $\mathrm{~N} \eta 2$ & & & & & & $3.0 \pm 0.2(21)$ & & \\
\hline & $\mathrm{O} 2$ & $\mathrm{R} 84$ & $\mathrm{~N} \varepsilon$ & & & & & & & $3.1 \pm 0.2(22)$ & \\
\hline & $\mathrm{O} 3$ & N82 & $\mathrm{O}$ & & & & & & & $2.8 \pm 0.1(68)$ & $2.8 \pm 0.2(49)$ \\
\hline & $\mathrm{O} 3$ & $\mathrm{R} 84$ & $\mathrm{~N} \varepsilon$ & & & & & & & $3.0 \pm 0.2(43)$ & \\
\hline & $\mathrm{O} 3$ & $\mathrm{R} 84$ & $\mathrm{~N} \eta 2$ & & & & & & & $3.0 \pm 0.2(37)$ & \\
\hline & O3 & $\mathrm{R} 84$ & $\mathrm{~N} \eta 1$ & & & & & & & & $3.0 \pm 0.2(39)$ \\
\hline
\end{tabular}

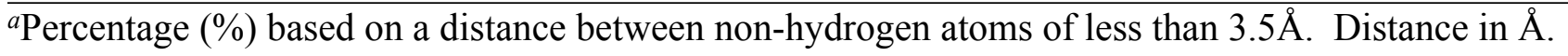


${ }^{b}$ When multiple hydrogen bonds are formed between two heavy atoms through different hydrogens, the occupancy of the interaction listed is the sum of all the individual hydrogen bonds and the distance is the average of all the individual hydrogen bonds.

${ }^{c}$ The occupancy of the interactions between two heavy atoms, calculated as the sum of all the individual hydrogen bonds through different hydrogens, is greater than $100 \%$. 

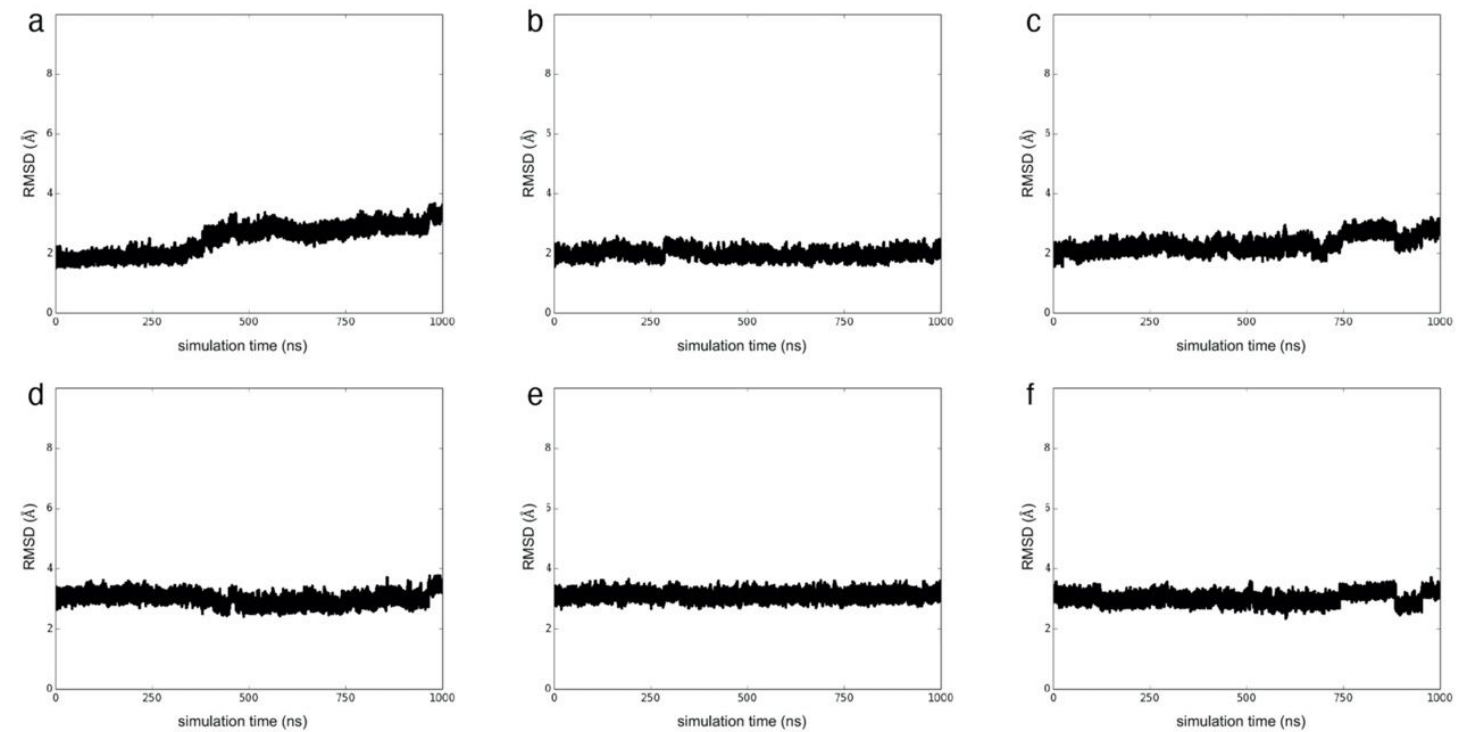

Figure S1. RMSD values for lectin domain during the course of three replicas of MD simulations ( $1: \mathrm{a}$ and $\mathrm{d} ; 2: \mathrm{b}$ and $\mathrm{e} ; 3: \mathrm{c}$ and $\mathrm{f}$ ) starting from the extended conformation. The reference structures are co-crystal complex (a, b, and c) and soak complex (d, e, and f). 

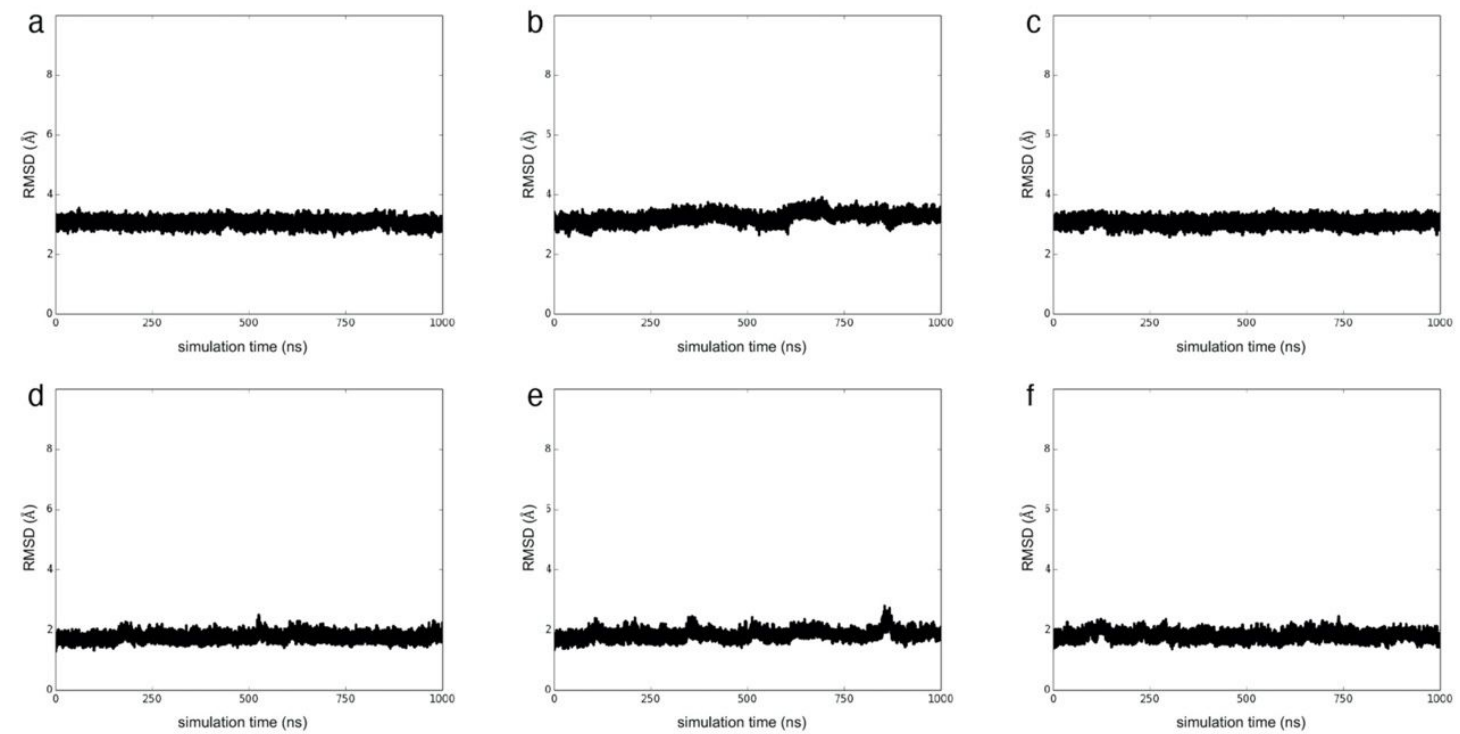

Figure S2. RMSD values for lectin domain during the course of three replicas of MD simulations (1: $a$ and $d ; 2: b$ and $e ; 3: c$ and $f$ ) starting from the bent conformation. The reference structures are co-crystal complex (a, b, and c) and soak complex (d, e, and f). 

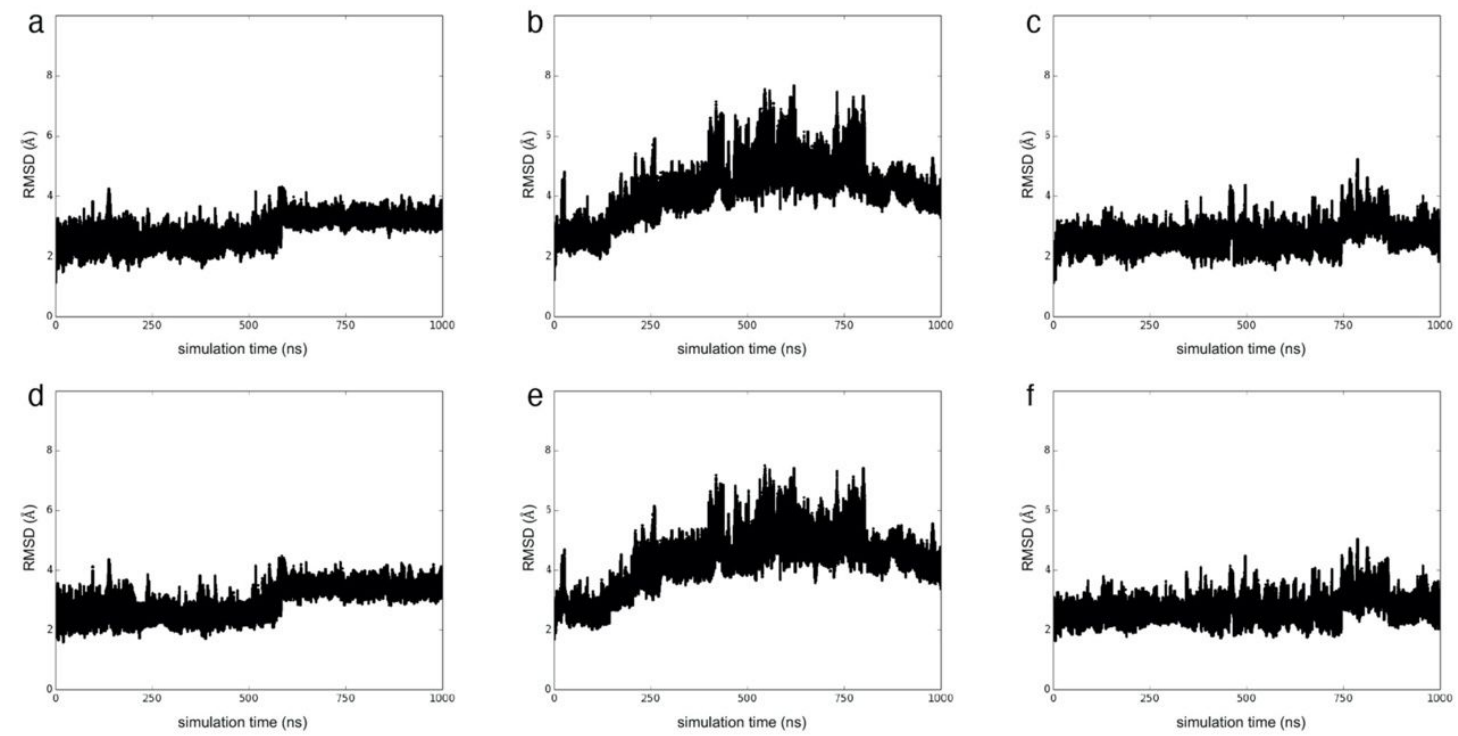

Figure S3. RMSD values for EGF domain during the course of three replicas of MD simulations (1: $a$ and $d ; 2: b$ and $e ; 3: c$ and $f$ ) starting from the extended conformation. The reference structures are co-crystal complex (a, b, and c) and soak complex (d, e, and f). 

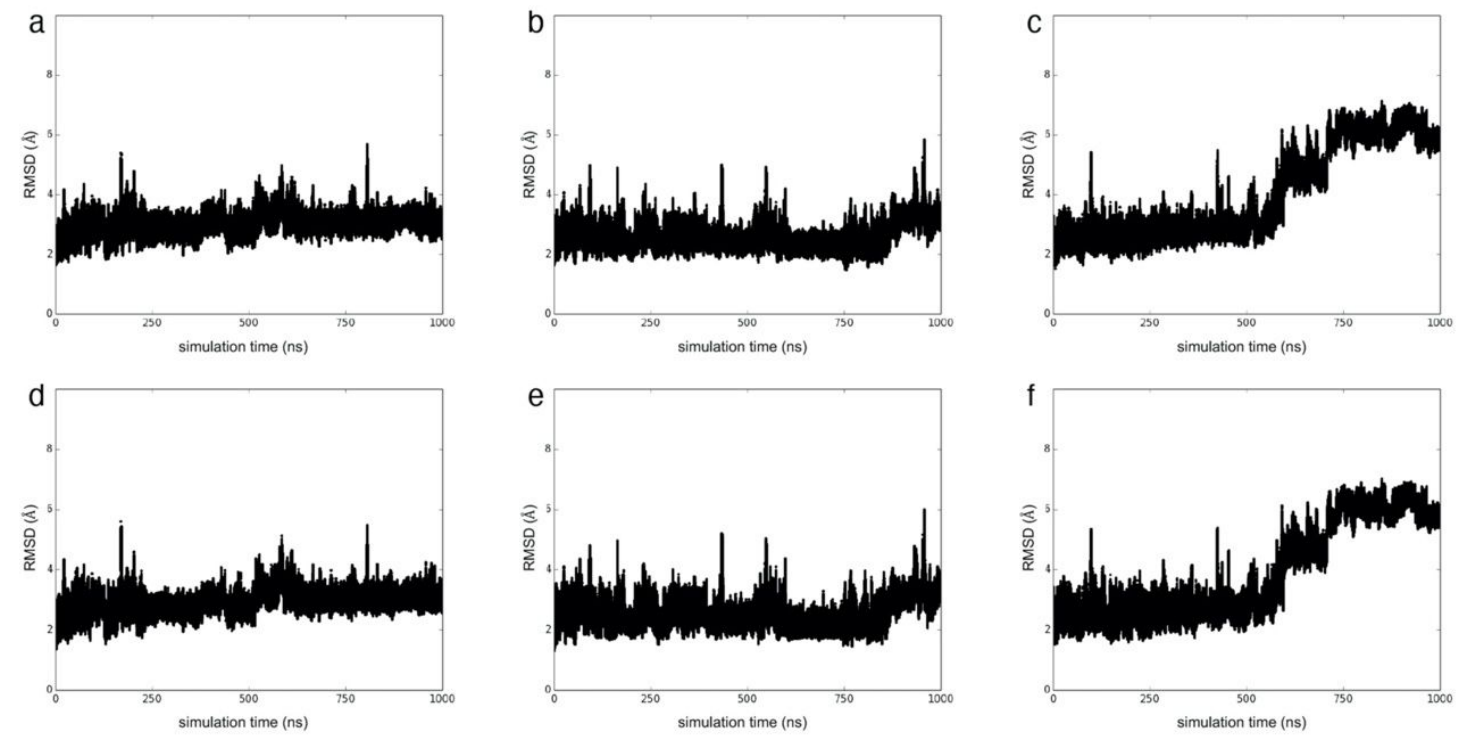

Figure S4. RMSD values for EGF domain during the course of three replicas of MD simulations (1: $\mathrm{a}$ and $\mathrm{d} ; 2$ : $\mathrm{b}$ and $\mathrm{e} ; 3$ : $\mathrm{c}$ and $\mathrm{f}$ ) starting from the bent conformation. The reference structures are co-crystal complex (a, b, and c) and soak complex (d, e, and f). 

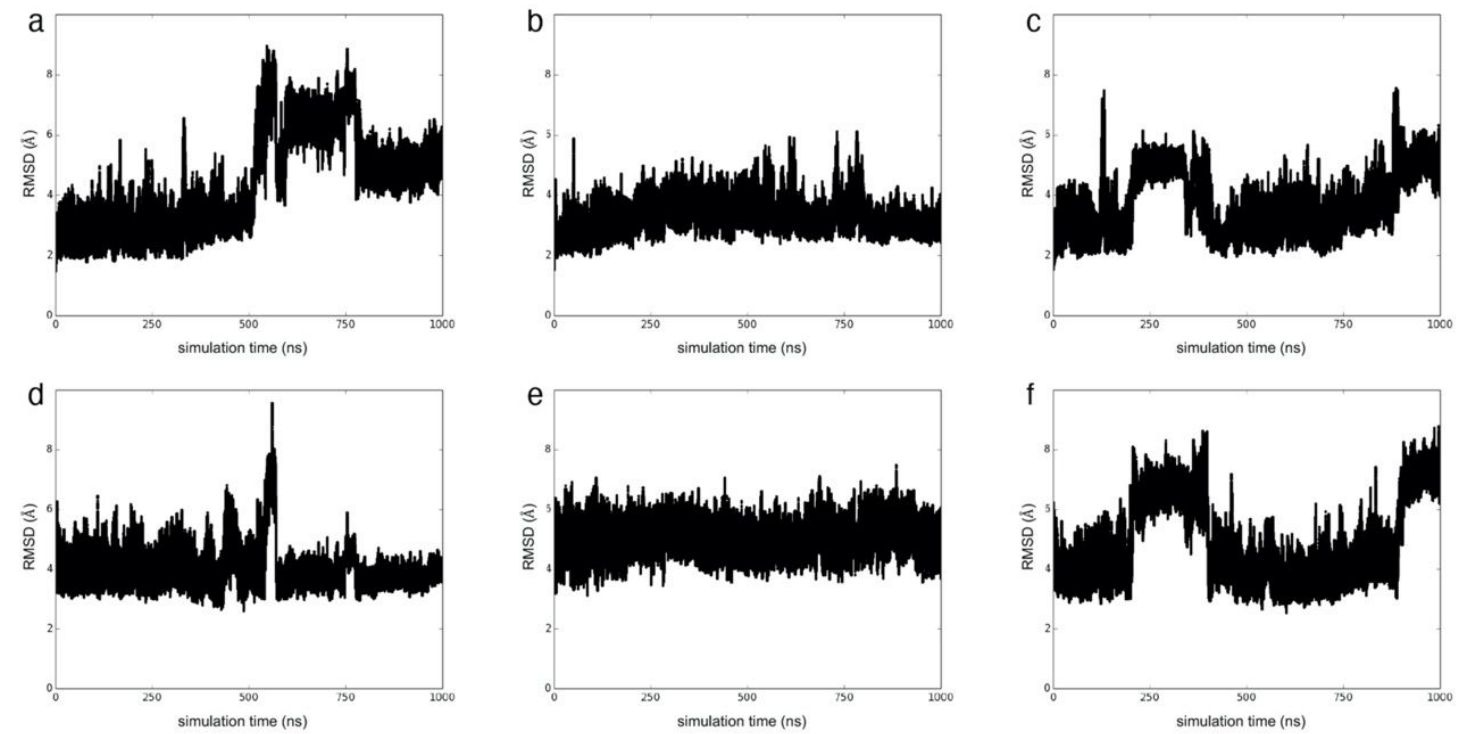

Figure S5. RMSD values for E-selectin during the course of three replicas of MD simulations (1: $\mathrm{a}$ and $\mathrm{d} ; 2$ : $\mathrm{b}$ and e; 3 : $\mathrm{c}$ and $\mathrm{f}$ ) starting from the extended conformation. The reference structures are co-crystal complex (a, b, and c) and soak complex (d, e, and f). 

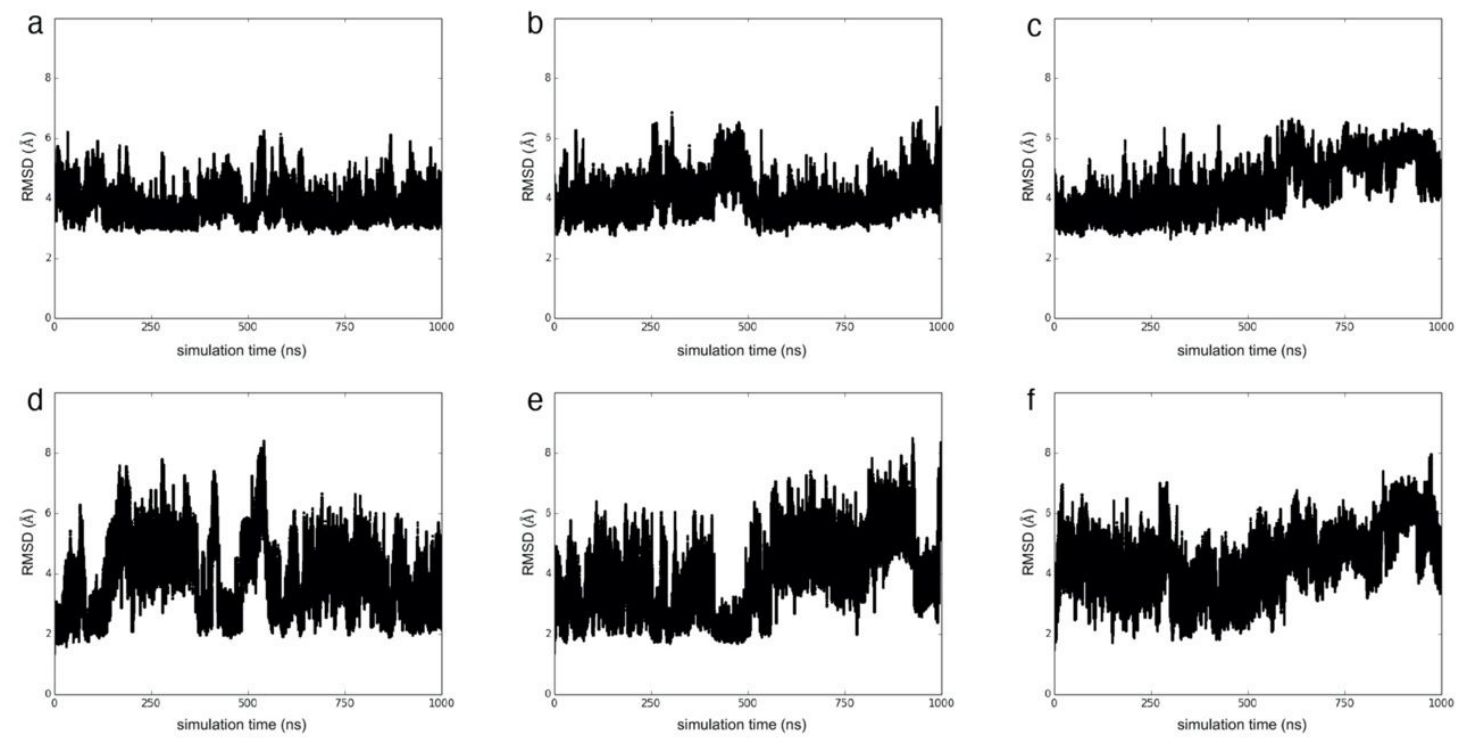

Figure S6. RMSD values for E-selectin during the course of three replicas of MD simulations (1: a and $d ; 2$ : $b$ and e; 3: $c$ and $f$ ) starting from the bent conformation. The reference structures are co-crystal complex (a, b, and c) and soak complex (d, e, and f). 

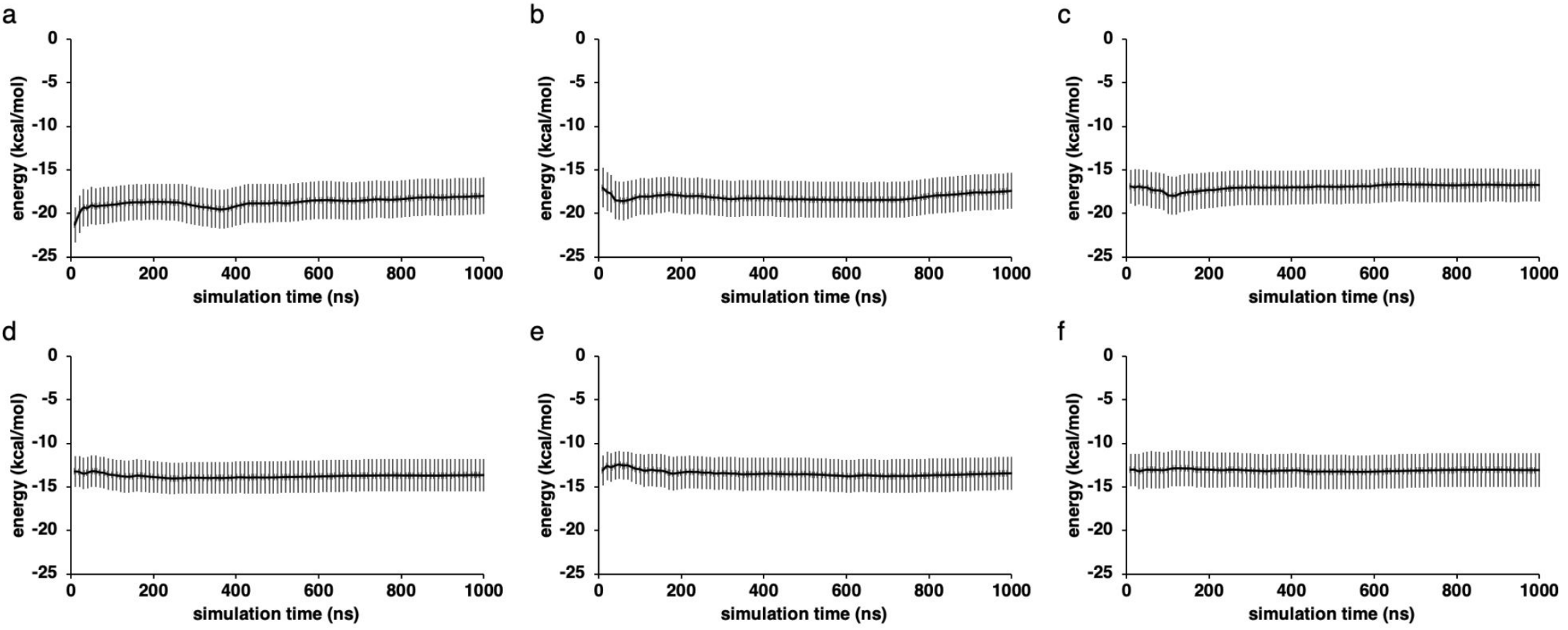

Figure S7. The running averages of MM-GBSA energies for E-selectin/sLe ${ }^{\mathrm{x}}$ complex calculated with trajectories from three replicas of MD simulations starting from co-crystal (a, b, and c) and soak (d, e, and f) complexes. 


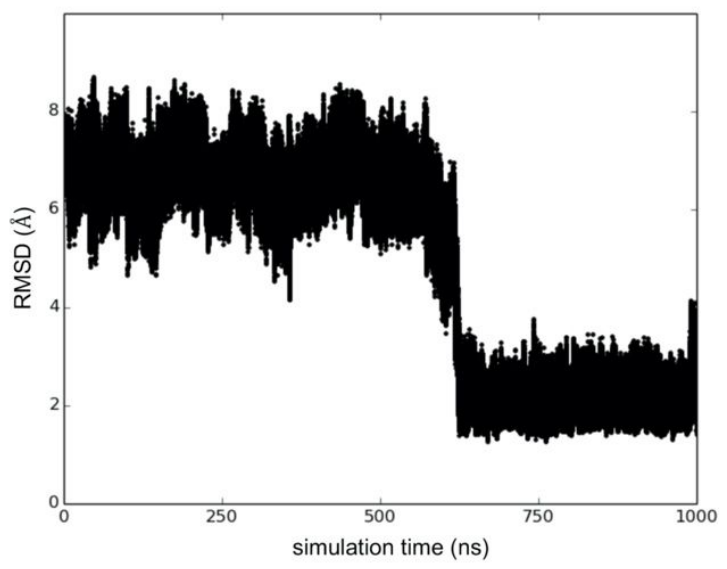

Figure S8. Positional RMSD values for residues in the loop by referencing to that in the close conformation. 


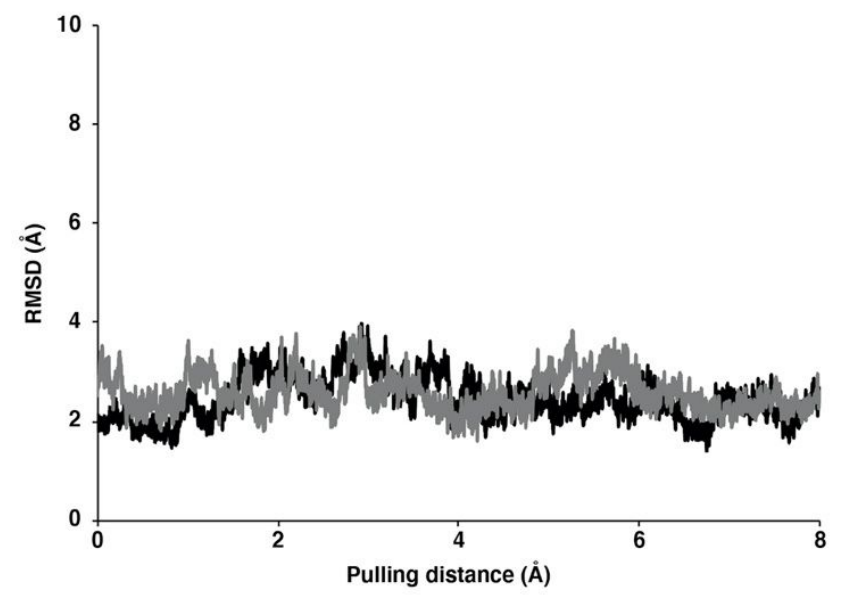

Figure S9. Positional RMSD values for residues in the loop residues during the course of ASMD simulations starting from co-crystal (black) and soak (grey) complexes. 